\title{
Beyond the diffraction limit
}

\author{
The emergence of imaging schemes capable of overcoming Abbe's diffraction barrier is revolutionizing \\ optical microscopy.
}

In 1873, the German physicist Ernst Abbe realized that the resolution of optical imaging instruments, including telescopes and microscopes, is fundamentally limited by the diffraction of light. His finding indicated that ultimately the resolution of an imaging instrument is not constrained by the quality of the instrument, but by the wavelength of light used and the aperture of its optics. This meant that a microscope could not resolve two objects located closer than $\lambda / 2 \mathrm{NA}$, where $\lambda$ is the wavelength of light and NA is the numerical aperture of the imaging lens.

This diffraction-limited phenomenon hindered the performance of optical microscopy for over a century, and was considered a fundamental, unbreakable rule. Recently, however, several new exciting approaches in imaging have emerged that can 'break' this rule under certain circumstances. It now appears that there is no fundamental limit in achieving spatial resolution; using visible light, it is possible to resolve up to a few nanometres with these approaches.

To celebrate these developments, this issue features a focus on super-resolution imaging techniques that operate beyond the diffraction limit. A collection of articles on different imaging techniques - from far-field fluorescence to plasmonics - can be found. The collection consists of a review article, a progress article, two commentaries and an interview.

In the interview on page 368 , W. E. Moerner highlights some of the incredible advantages of imaging when it is not limited by diffraction ${ }^{1}$. Such techniques not only allow non-invasive investigation of the interior of biological cells, but also promise nano-imaging of semiconductor devices in the electronics industry. Moerner elegantly summarizes the advantages and disadvantages of the various subdiffractionlimited imaging approaches developed to date, and also discusses the future outlook of such techniques.

When reading these articles, you will find that the techniques can be classified into several groups. First, there are near- and farfield approaches: the former operate close to the sample, often collecting evanescent signals that decay rapidly but contain extra information about the sample, whereas the latter collects optical signals (typically

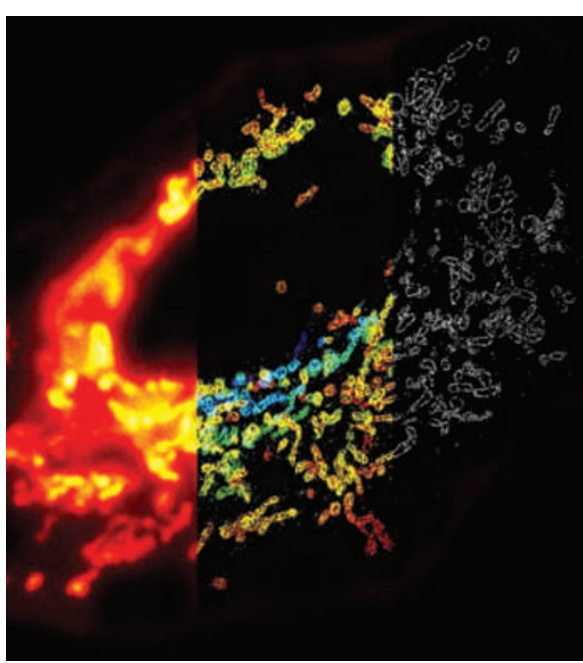

to achieve high-resolution fluorescence imaging - now allows imaging of cell interiors to unprecedented levels of detail. On page 365, Xiaowei Zhuang explains how a spatial resolution of a few tensof-nanometres can be achieved using this approach ${ }^{3}$. She also describes how multicolour, three-dimensional stochastic optical reconstruction microscopy can be put to good use for imaging molecular structures in cells.

On page 362, Rainer Heintzmann and Mats Gustafsson take a broader look at the problem of subdiffraction imaging and discuss what is necessary to make it possible. They explain how the use of a spatially non-uniform illumination and a sample with a nonlinear photoresponse are the only two fundamental requirements for superresolution imaging of a spatially continuous fluorescence distribution ${ }^{4}$.

Aside from fluorescence, surface plasmons (those that are either excited on the metallic surface or localized in a small metallic tip) also provide a method of overcoming the diffraction limit of light. The review article by Satoshi Kawata and co-workers on page 388 comments on the potential for plasmonics to improve imaging 5 .

As Moerner explains in the interview, all these different approaches that image beyond the 'diffraction limit' are complementary. One thing is clear: the achievements of super-resolution imaging are narrowing the gap between optical microscopy and ultrahigh resolution imaging schemes (such as X-ray or electron microscopy), which rely on much shorter wavelengths than visible light. The arrival of super-resolution optical microscopes will make it possible to observe the world around us with a new level of clarity. This will almost certainly result in some very exciting new science, particularly within - but not limited to - the field of biology.

\footnotetext{
References

1. Won, R. Nature Photon. 3, 368-369 (2009).

2. Stefan, W. H., Schmidt, R. \& Egner, A. Nature Photon. 3, 381-387 (2009).

3. Zhuang, X. Nature Photon. 3, 365-367 (2009).

4. Heintzmann, R. \& Gustafsson, M. G. L. Nature Photon. 3, 362-364 (2009).

5. Kawata, S., Inouye, Y. \& Verma, P. Nature Photon. 3, 388-394 (2009).
} 